\title{
The Indirect Cost Burden of Cancer Care in Canada: A Systematic Literature Review
}

\author{
Nicolas Iragorri ${ }^{1,2,4}$ C $\cdot$ Claire de Oliveira ${ }^{1,2,3} \cdot$ Natalie Fitzgerald $^{1} \cdot$ Beverley Essue ${ }^{1,2}$
}

Accepted: 21 October 2020 / Published online: 24 December 2020

(c) The Author(s) 2020

\begin{abstract}
Background and objectives Cancer poses a substantial health and economic burden on patients and caregivers in Canada. Previous reviews have estimated the indirect cost burden as work-related productivity losses associated with cancer. However, these estimates require updating and complementing with more comprehensive data that include relevant dimensions beyond labor market costs, such as patient time, lost leisure time and home productivity losses.

Methods A systematic review of the literature was conducted to identify studies published from 2006 to 2020 that measured and reported the indirect costs borne by cancer patients and their caregivers in Canada, from the patient, caregiver, employer, and societal perspectives. Study characteristics and cost estimation methods were extracted from relevant studies. Costs estimates were reported and converted to 2020 CAD for the following categories: lost earnings, caregiving time costs, home production losses, patient time (leisure), morbidity-, disability-, premature mortality-related costs, friction costs, and overall productivity losses. A quality assessment of individual studies was conducted for included studies using the Newcastle-Ottawa Assessment Tool.

Results In total, 3980 studies were identified, of which 18 Canadian studies met the inclusion criteria for review. One-third of the studies used or developed prediction models, $38 \%$ enrolled patient cohorts, and $27 \%$ used administrative databases. Over one-third of the studies were conducted at a national level (38\%). All studies employed the human capital approach to estimate costs, and 16\% also used the friction cost approach. Lost earnings were higher among self-employed patients (43\% vs $24 \%$ among employees) and females ( $\$ 8200$ vs $\$ 3200$ for males). Caregiver costs ranged from $\$ 15,786$ to $\$ 20,414$ per patient per year. Household productivity losses were estimated to be up to $\$ 238,904$ per household per year. Patient time (leisure) costs were estimated to be between $\$ 13,000$ and $\$ 18,704$ per patient per year. Premature annual mortality costs were estimated to be $\$ 2.98$ billion overall in Quebec. Friction costs incurred by employers were estimated between $\$ 6400$ and $\$ 23,987$ per patient per year. Societal productivity losses associated with cancer were estimated between $\$ 75$ million to $\$ 317$ million, annually.

Conclusions This review suggests that the indirect cost burden of cancer is considerable from the patient, caregiver, employer, and societal perspectives. This up-to-date review of the literature provides a comprehensive understanding of the indirect cost burden by including non-labor market activity costs and by examining all relevant perspectives. These results provide a strong case for the government and employers to ensure there are supports in place to help patients and caregivers buffer the impact of cancer so they can continue to engage in productive activities and enjoy leisure time.
\end{abstract}

Electronic supplementary material The online version of this article (https://doi.org/10.1007/s40258-020-00619-z) contains supplementary material, which is available to authorized users.

Nicolas Iragorri

nicolas.iragorri@mail.utoronto.ca

Extended author information available on the last page of the article 


\section{Key Points for Decision Makers}

Cancer patients, their caregivers, and employers bear a considerable indirect cost burden related to cancer care in Canada.

The indirect cost burden of cancer is not limited to the productive labor market costs incurred by patients. Patient leisure time and home-production losses are important cost categories that must be considered when measuring the indirect cost burden.

The caregivers of pediatric patients, women, younger patients, and those who were self-employed face higher indirect costs in Canada.

\section{Introduction}

Cancer is a common chronic disease that has a large impact globally, including in Canada. Around half of the population is expected to develop cancer in their lifetime [1], and it remains the leading cause of premature mortality [2]. Furthermore, cancer poses a substantial health and economic burden on patients, their caregivers, and the healthcare system, given its high incidence (over 200,000 cases per year in 2019), long-term health effects, and rising treatment costs [3].

The economic burden is typically conceptualized as three distinct categories: direct, indirect, and psychosocial [4]. Cost of illness studies are often conducted to estimate the direct and indirect costs associated with a given disease; in turn, these estimates are used to support cost-effectiveness analyses, which are critical for informing resource allocation decisions [5]. Although the direct burden of cancer in Canada has been previously described [6], evidence around the indirect costs associated with cancer care is sparse and limited. The indirect burden includes the monetary losses associated with lost patient/caregiver time and lost opportunities due to disease morbidity and related premature mortality (also defined as opportunity costs) [7]. These costs are incurred by patients, caregivers, employers, and society as a whole; however, because lost opportunities are usually not reflected in monetary transactions, the value of the time lost must be approximated. Thus, to obtain an overall estimate of the indirect cost burden, the time that cancer patients spend in obtaining treatment, loss from not working due to shortor long-term cancer-related disability, and the lost productivity due to premature death are monetized and combined [7].
In a report conducted by the Public Health Agency of Canada, work-related productivity losses due to cancer were estimated to be $\$ 586,000,000$ in 2008 [8]. Subsequently, a review conducted in 2010 identified studies published before 2008 that had estimated wage losses due to cancer [9] and found that newly diagnosed cancers in Canada generated an average wage loss of $\$ 3.18$ billion per year. However, these studies only included labor market-related production losses. Considering the lack of up-to date and comprehensive estimates, and the fast pace at which cancer care has evolved in the last decade [10], the evidence around the indirect cost burden of cancer in Canada needs to be re-evaluated to include recent studies that also capture non-labor market activities, such as home production, leisure, and caregiving time. Therefore, the aim of this review was to evaluate the most recent Canadian literature on the indirect cost burden associated with cancer from the perspectives of patients, families, caregivers, employers, and society.

\section{Methods}

\subsection{Data Sources and Search Strategies}

A systematic review of the literature was conducted to identify studies that estimated and/or reported on the indirect cost burden of cancer in Canada. We searched MEDLINE, EMBASE (Excerpta Medical Database), CINAHL, Econlit, PsychINFO, Cochrane, and Erudit (this last to capture literature published in French). We also searched Open Gray to account for relevant gray literature. All databases were searched between January 1, 2006 and January 8, 2020. Search terms combined medical subject headings (MeSH), Embase subject headings (Emtree), and keywords for cancer (e.g. oncology), economic burden (e.g. costs), and indirect costs (e.g. productivity loss). In addition, we searched the reference lists of all included studies to identify additional relevant studies. The full search strategy and key words can be found in Appendix 1. We followed the Preferred Reporting Items for Systematic Reviews and Meta-Analyses (PRISMA) guidelines [11] as presented in Appendix 2.

\subsection{Eligibility Criteria}

We included any study that reported and/or estimated the indirect cost burden of cancer patients and/or their caregivers. No study design or language restrictions were applied to the search. Studies were excluded if at least one of the following criteria was met: studies evaluated non-cancer populations, only reported or measured costs that were not defined as indirect costs (e.g. out-of-pocket), were not fulltext articles (e.g. conference abstracts), or were conducted without employing Canadian data. After running the search, 
the identified records were screened by title and abstract, followed by a full-text review by two independent reviewers (NI and BE). Any article that either reviewer included at the title/abstract review stage was included for full-text review. Disagreements at the full-text stage were settled by discussion until a consensus was reached with a third reviewer $(\mathrm{CdO})$.

\subsection{Data Extraction}

We extracted the following data from the included studies: authors' names, title of the study, year of publication, type of publication (e.g. article, report), jurisdiction (e.g. federal-, province- or territory-level analysis), cancer site, cancer care continuum (from diagnosis to end-of-life care), study population, type of study (e.g. prospective), sample size, mean age of population examined, proportion of females reported in the study, employment status, income level, control/comparison group, and databases/sources used. Regarding the outcome of interest, we extracted data on the definition of the indirect cost, methods, and tools (e.g. surveys) used to measure the indirect costs, cost estimates, currency, currency year, and time frame or recall period. Costs were reported and divided by different categories: (1) lost earnings (at the patient level), (2) caregiving time costs; (3) home production losses, defined as production activities within the household, which are usually not remunerated (e.g. cleaning). Caregiving costs and home production losses were reported separately, as caregiving might not always be delivered at home; (4) lost patient time (i.e. lost leisure time) (5) morbidity-, disability-, and premature mortality-related costs (productivity losses and time lost due to cancer-related disability and mortality); (6) friction costs (costs that employers incur when replacing absentees); and (7) aggregated productivity losses from a societal perspective.

\subsection{Quality Assessment}

Quality assessment was ascertained using the NewcastleOttawa Assessment Tool [12] and conducted by two independent reviewers (NI and BE). Prospective and retrospective studies were evaluated with specific versions adapted to cohort and cross-sectional study designs, respectively [12]. Three domains were evaluated for all prospective and retrospective studies: selection (i.e. representativeness of the sample), comparability (i.e. comparability of subjects, inclusion of confounding factors), and outcome (i.e. assessment of outcome, statistical test used). A 'star system' was used to judge the extent to which each individual study accounted for issues concerning these three categories. Each domain was assessed for risk of bias (unclear, low, or high) according to the total score assigned and the pre-specified and validated thresholds identified by the tool developers. For retrospective studies, the maximum scores for the Selection, Comparability and Outcome domains were 5, 2, and 3 stars, respectively. Studies were identified as having low risk of bias if their overall score was 8 or higher. Studies with an overall score below 5 were identified as having a high risk of bias. For prospective studies, the maximum scores for the Selection, Comparability and Outcome domains were 4, 2, and 3 stars, respectively. Studies were identified as having low risk of bias if the Selection, Comparability, and Outcome domains scored at least 3,1 , and 1 star(s), respectively. Studies were identified as having high risk of bias if the Selection domain scored less than 2 stars, or if the Comparability or Outcomes domains scored no stars. Quality assessment was not conducted for studies that used predictive models based on incidence cost approaches.

\subsection{Evidence Synthesis}

We provided a description of the different types of costs reported in the literature and conducted a narrative synthesis of the estimation methods employed. Indirect costs were reported as an annual expenditure, and converted to 2020 Canadian dollars (CAD) using the Consumer Price Index from the Bank of Canada Inflation Calculator [13]. A meta-analysis was not undertaken due to the heterogeneity of the patient populations and cost definitions. Therefore, we undertook a narrative synthesis of the literature.

\section{Results}

\subsection{Summary of Studies}

Searches on the electronic database identified 3980 records, of which 3009 were unique (971 duplicates); 403 were included for full-text review. Among the 403 studies, 18 Canadian studies were ultimately retained [14-31]. The results of the electronic searches, the eligibility criteria and the reasons for exclusion are presented in Fig. 1. Over half of the excluded records (62\%) in the title/abstract review stage reported costs that were not relevant (e.g. direct costs), and $18 \%$ of the full-text studies failed to explicitly report or measure indirect costs. Non-Canadian studies were only excluded during the full-text review $(n=151)$.

The study characteristics of the included studies are summarized in Table 1. The combined sample size of the 18 studies was 550,501 cancer patients and/or caregivers and ranged from 50 to 196,050 individuals. Studies with large samples usually identified cases through the linkage of various administrative datasets, such as the Canadian Cancer Registry, census data, the Labour Force Survey, the Canadian Community Health Survey, and the tax files. A total of 547,162 cancer patients were identified through 


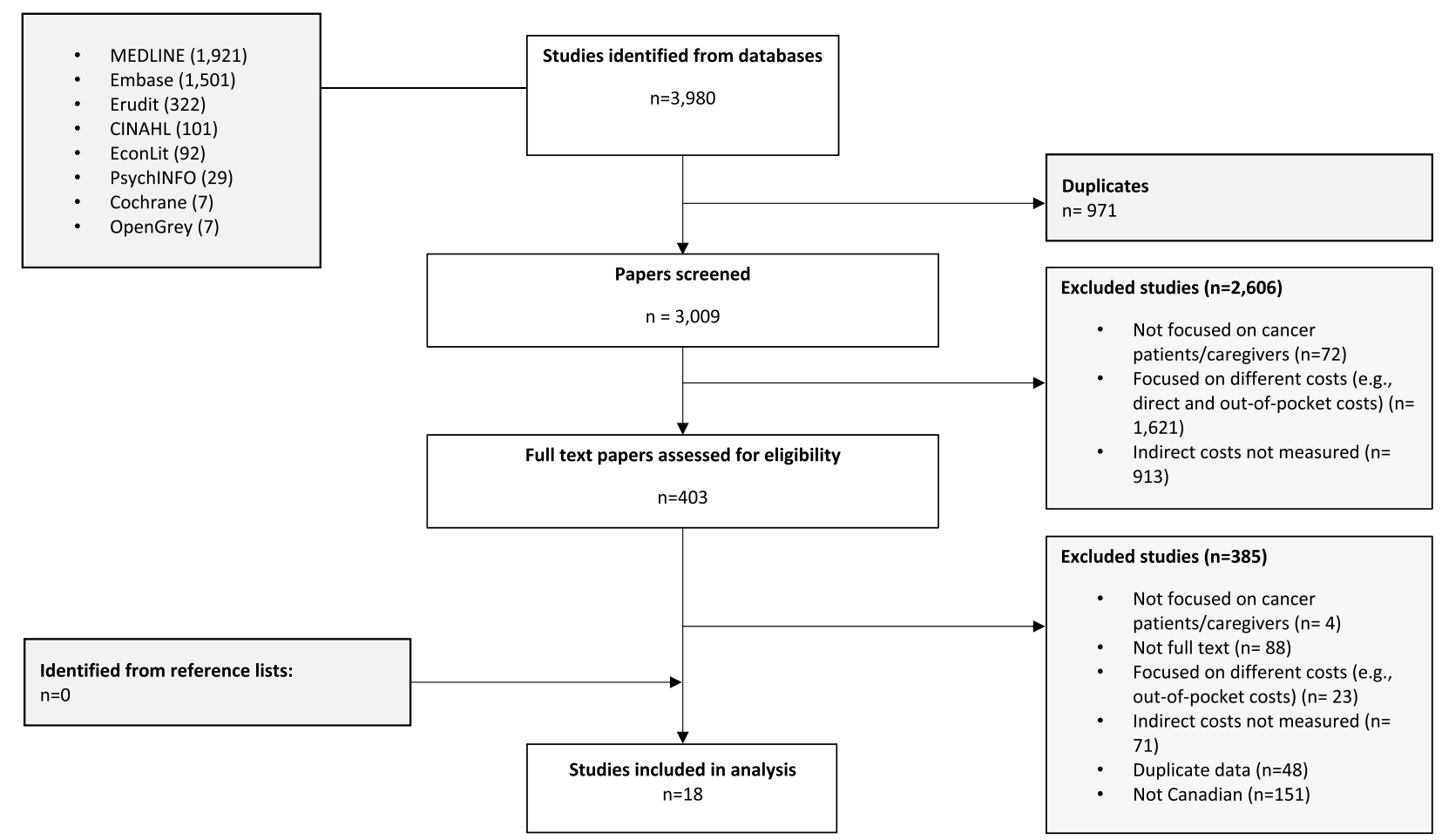

Note: This diagram shows the flow of information through the different sections of the systematic review, including the identified, excluded and included studies after the title/abstract and full-text reviews.

Fig. 1 Preferred reported items for systematic reviews and meta-analyses (PRISMA) diagram

these databases. Cohort studies were usually smaller due to the elevated costs associated with enrolling and following patients over time and collecting data on their incurred indirect costs; a total of 1962 patients and 1377 caregivers were enrolled in the prospective studies. Seven studies were prospective (38\%), five were retrospective (28\%) and 6 used mathematical models (33\%) to predict cancer cases and estimate the indirect cost burden. The papers with predictive models employed Markov chains [19], population attributable fractions [20, 25], and incidence-based approaches using data from cancer registries $[14,21,26]$. The publication years ranged from 2006 to 2018. Seven studies (38\%) reported on the indirect cost burden of cancer at the national level, while 6 (33\%) focused exclusively on Ontario, 3 (17\%) on Quebec, and $1(5 \%)$ on Nova Scotia. One study (5\%) evaluated the indirect cost burden on multiple provinces (Manitoba, Quebec, and Nova Scotia). Regarding patient populations, half evaluated multiple cancer types. Among these, two focused exclusively on pediatric populations. The remaining studies estimated costs for individual cancer sites, including breast, bladder, colorectal, lung, prostate, lung, mesothelioma, and melanoma. The mean age of the nonpediatric populations ranged from 48 to 73 years of age, while the mean age for the pediatric populations was 7.8 years. The percentage of females was close to $50 \%$ for most studies. Finally, half of the studies evaluated the indirect burden across the entire cancer care continuum, from diagnosis to palliative and end-of-life care. Three studies (16\%) focused exclusively on patients undergoing cancer treatment; two (11\%) on palliative and end-of-life care, and two (11\%) on survivorship.

\subsection{Indirect Costs}

The reported indirect costs were extracted and divided into the following categories: lost earnings, caregiver time costs, home production losses, patient leisure time costs, premature death/disability costs, friction costs, and societal productivity losses:

\section{Lost earnings}

The estimates of the indirect costs were reported by category and presented in Table 2. Further information regarding the cost definitions and methods of estimation for each study is summarized in Appendix 3. The most commonly reported indirect costs were lost earnings for cancer patients and their caregivers. After transforming all estimates to annual costs, the average wage loss ranged from $\$ 4538$ (in $2020 \mathrm{CAD}$ ) for the members of the families' support networks who resided outside the families' 


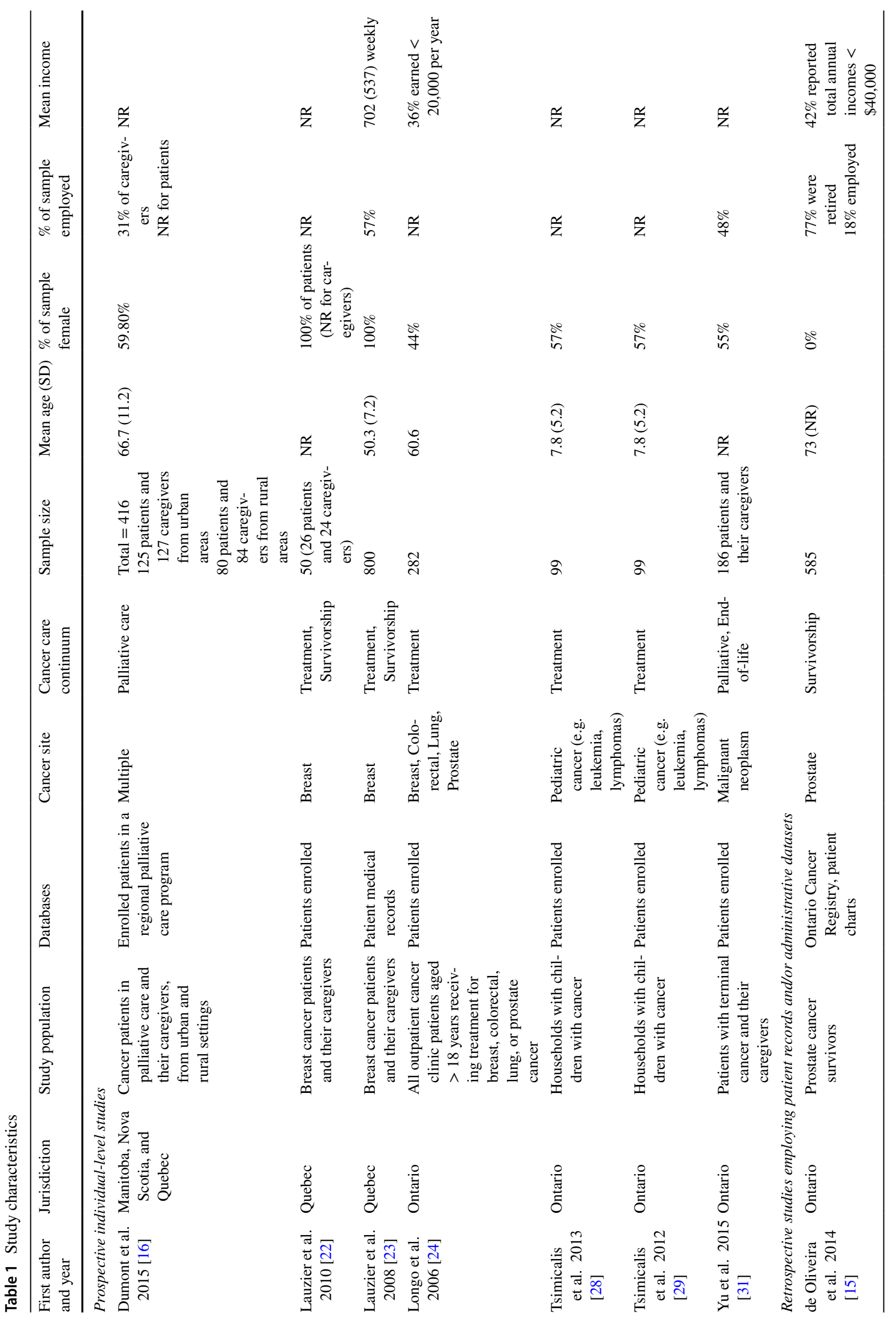




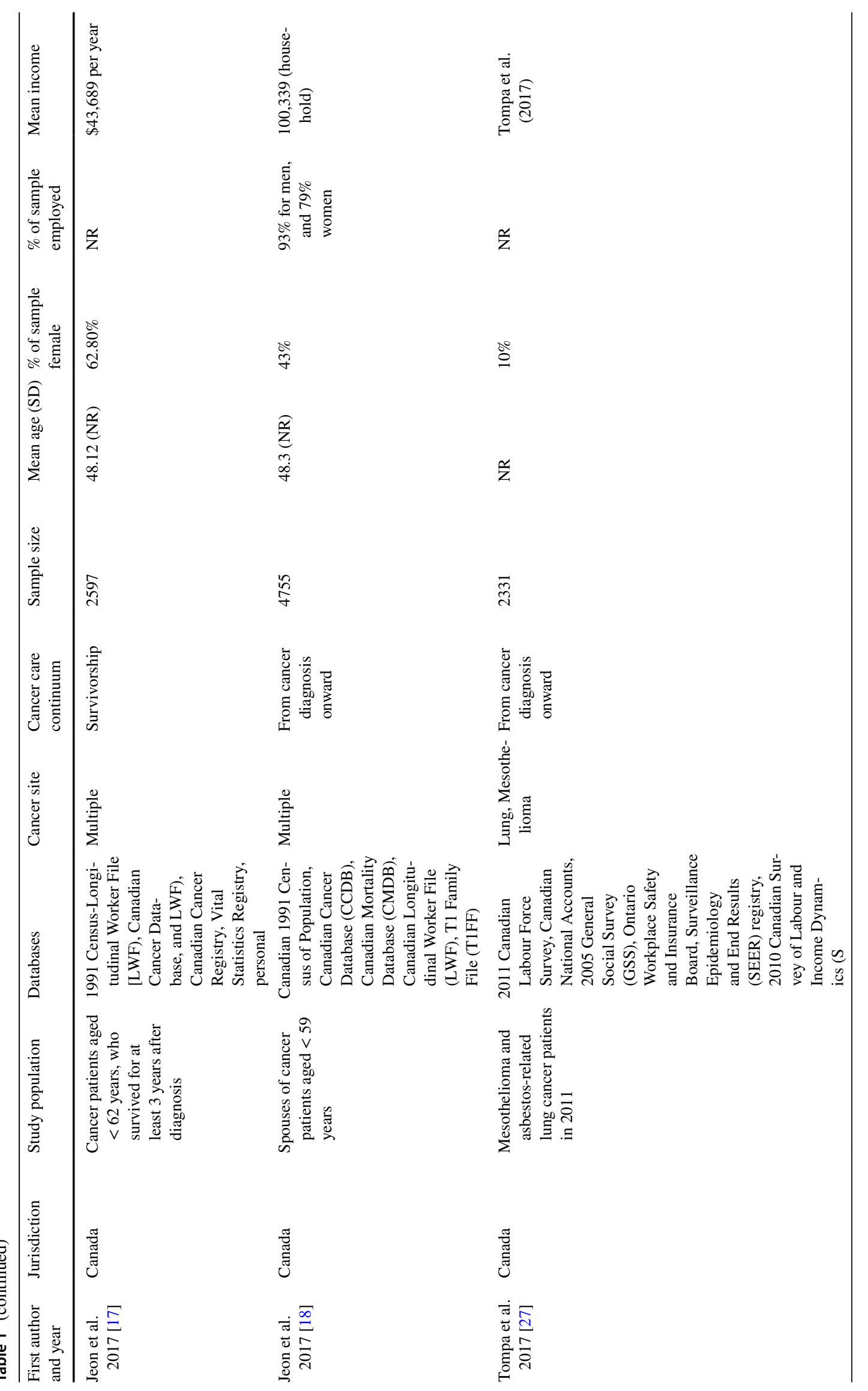




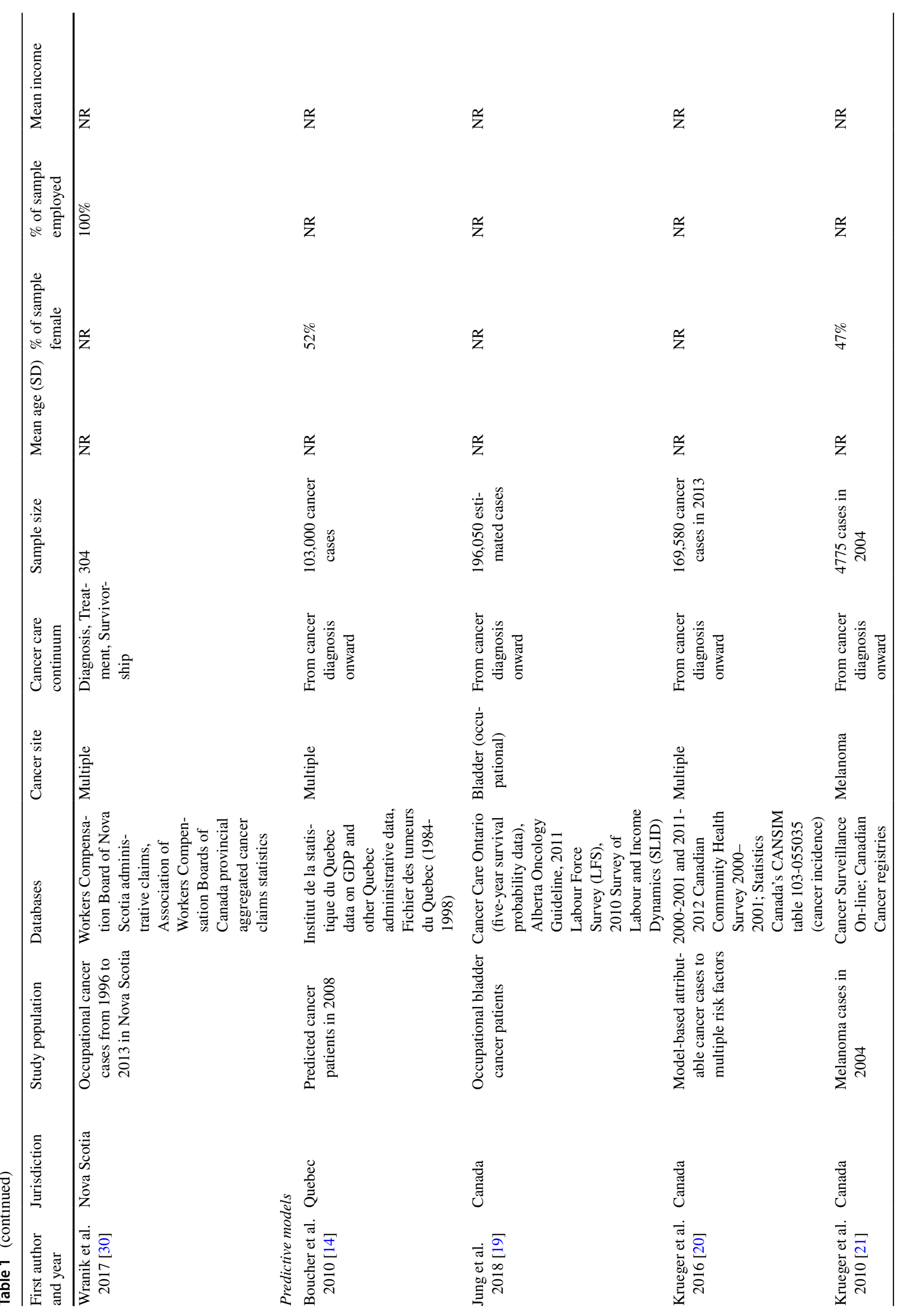




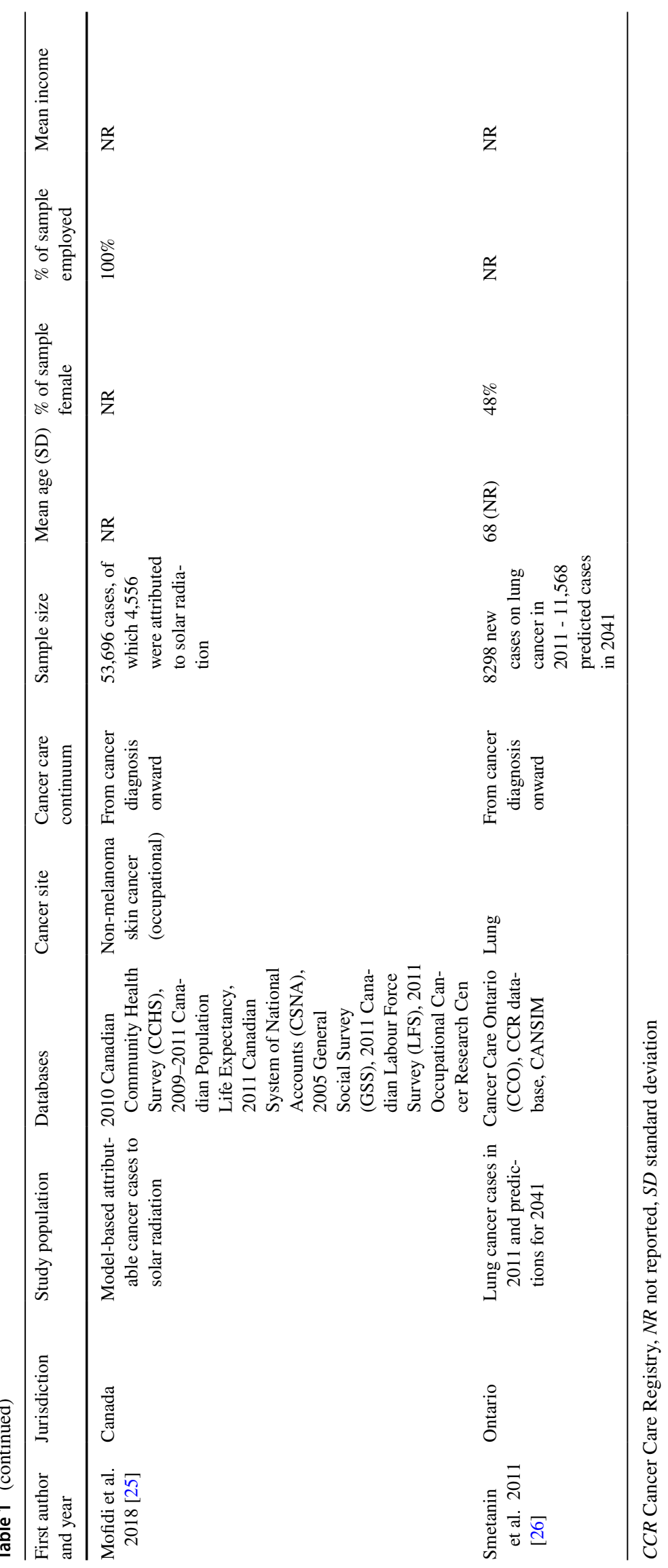




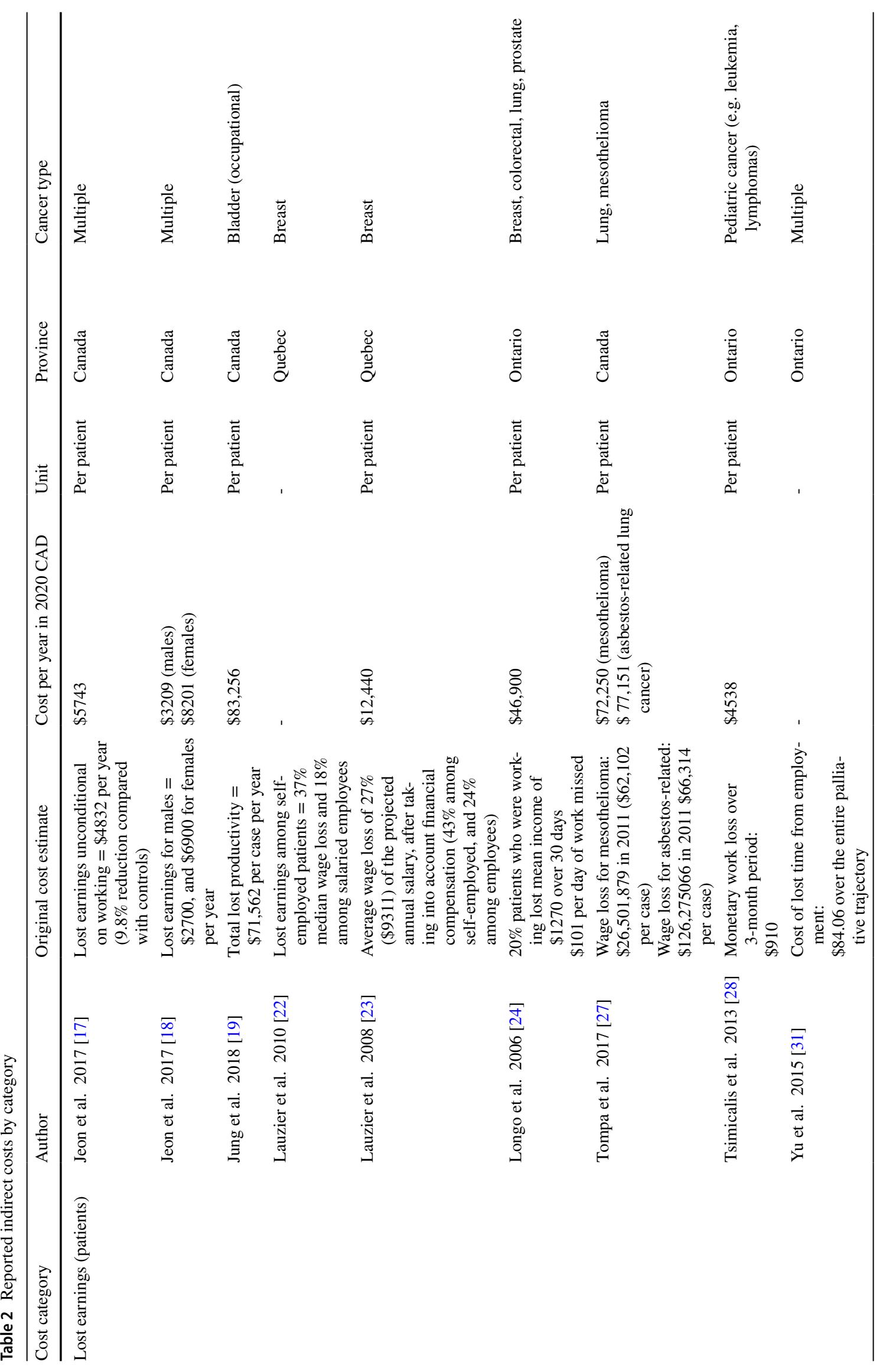




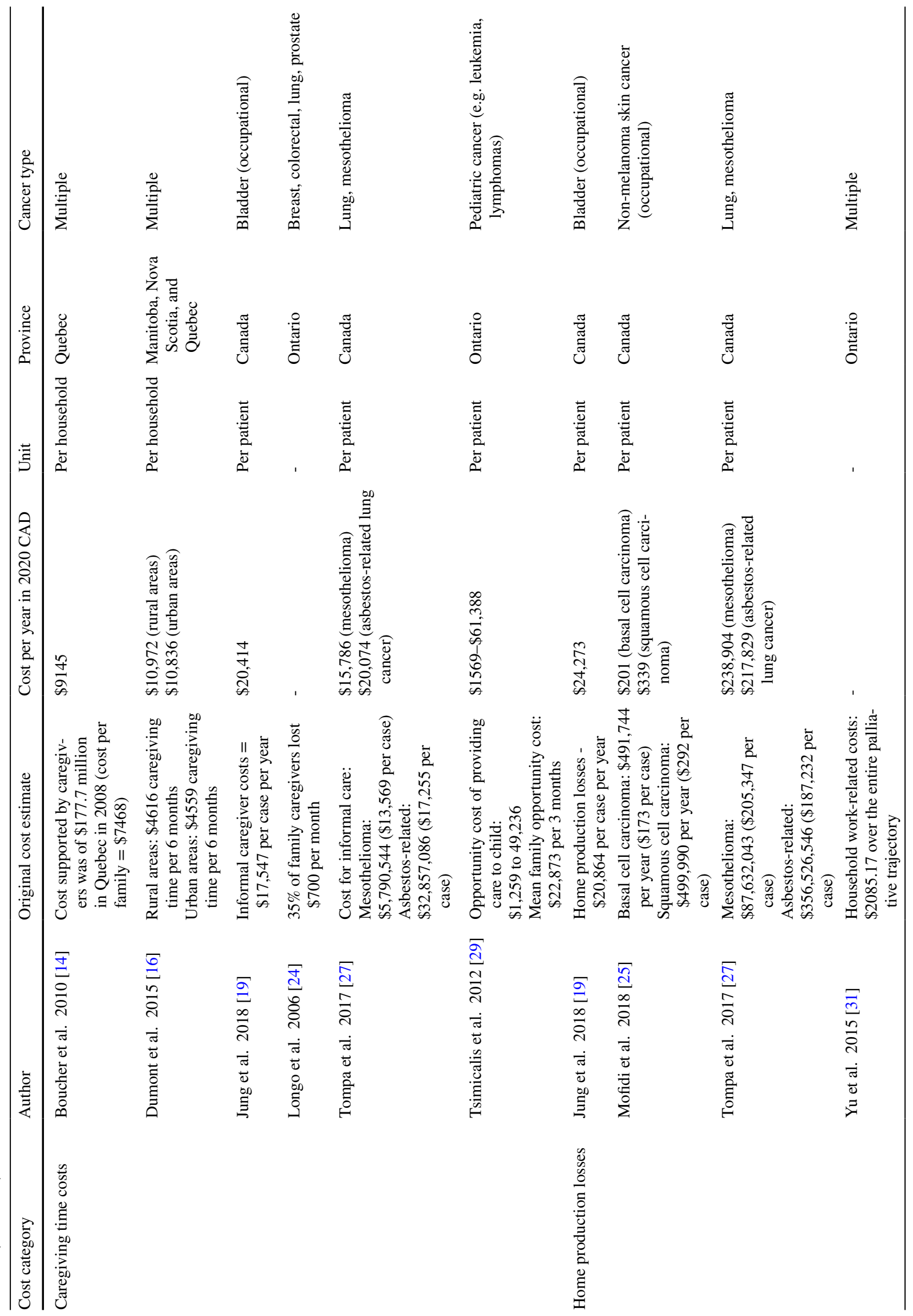




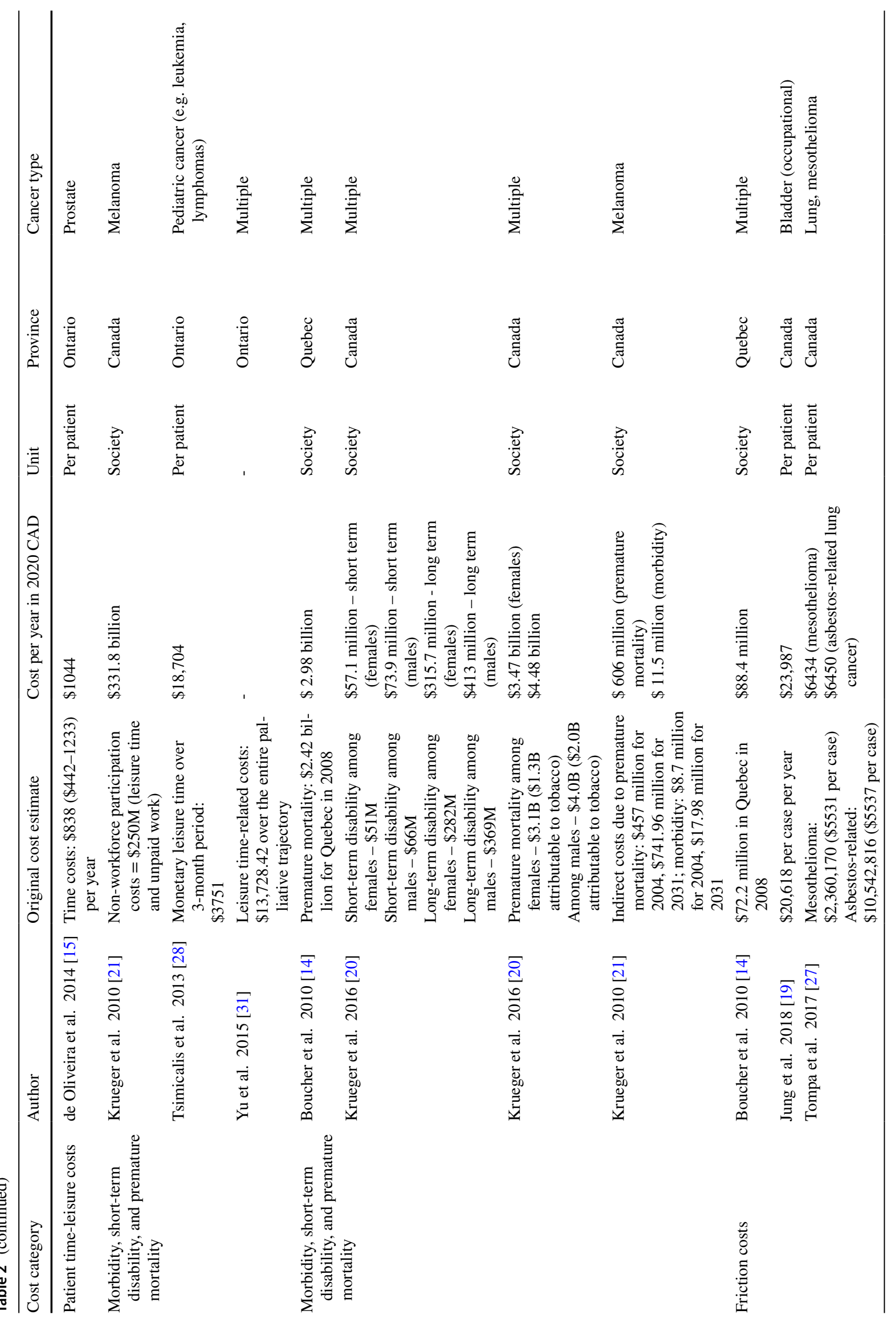




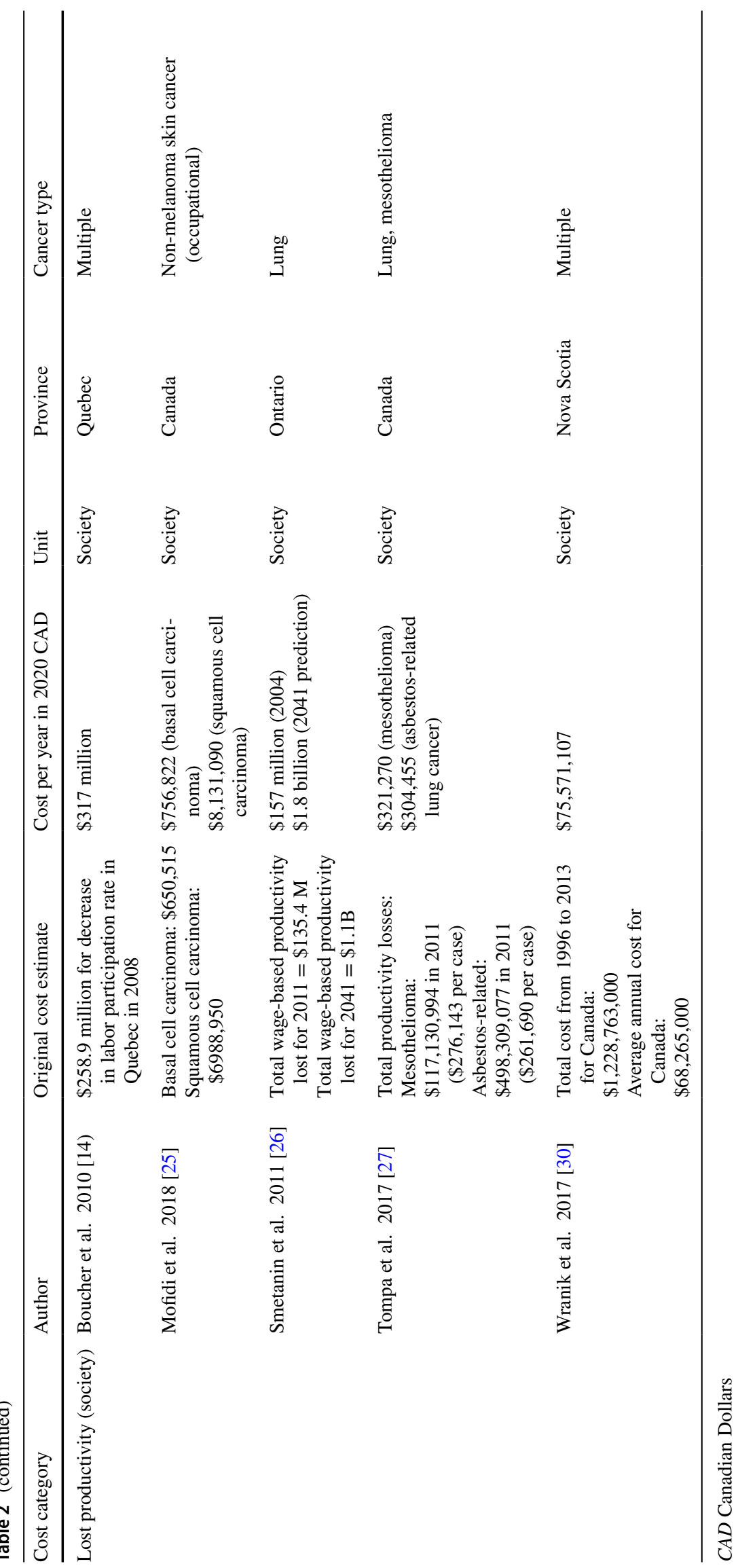


home in Ontario [28] to $\$ 83,256$ among patients with bladder cancer nationwide [19]. The evidence suggests that self-employed patients faced higher earning losses compared to salaried patients. Two studies conducted in Quebec estimated that self-employed patients with breast cancer experienced a wage loss of $48 \%$ and $37 \%$ of their projected salary, compared with $24 \%$ and $18 \%$ among salaried patients, respectively [22, 23]. On the other hand, a study that used a prediction model to calculate the productivity losses of the spouses of cancer patients estimated higher annual mean costs among females compared to males (\$8201 and $\$ 3209$, respectively) [18].

2. Caregiver time costs

Caregiver time costs were reported to account for non-labour market-related costs. Six studies reported a monetary value associated with the time that people spent caring for cancer patients. The average annual cost ranged from $\$ 15,786$ among patients with mesothelioma [27] to $\$ 20,414$ for patients with bladder cancer nationwide [19]. One study estimated average caregiver time costs, which ranged from $\$ 1569$ to $\$ 61,388$ for parents caring for a child with cancer in Ontario [29]. Another study conducted in Manitoba, Nova Scotia, and Quebec estimated that caregivers from rural and urban settings faced similar average annual indirect costs related to informal care ( $\$ 10,972$ and $\$ 10,836$, respectively) [16].

3. Home production losses

Home production losses are a different type of nonmarket activity costs, which pertain to production activities within the household, such as cooking and childcare, and that do not usually involve a monetary transaction. Home production losses were estimated using an average hourly wage for housekeepers and related occupations. Therefore, home production was often measured as zero for terminal cases due to the inability to work at home. Four studies reported home production losses, with annual average estimates ranging from $\$ 201$ for patients with basal cell carcinoma (skin cancer) [25] to $\$ 238,904$ among patients with mesothelioma [27]. Average household productivity costs were estimated at around \$2500 per patient per year in Ontario [31].

4. Patient leisure time costs

Patient leisure time costs were estimated in four studies (three conducted in Ontario and one at the national level) by determining the total personal/leisure time lost by patients and their caregivers due to cancer. As an example, de Oliveira et al. (2016) defined these costs as the time taken by patients and an accompanying person to visit health professionals as well as lost leisure time [15]. This time spent seeking care is considered an opportunity cost, as it could have been used to work or spent on different productive or non-productive activities (e.g. leisure). Although these estimates are not nec- essarily related to the labour market, all were estimated using an average hourly wage to assign a monetary value to the time lost. Krueger et al. estimated total nationwide costs related to lost leisure time and unpaid work as a result of cancer to be around $\$ 331$ billion per year [21]. Tsimicalis et al. estimated lost leisure time of $\$ 18,704$ per year per parent who cared for children with cancer in Ontario [28]; Yu et al. estimated a similar cost of $\$ 13,000$ per year per cancer patient [31].

5. Premature death/disability costs

Premature mortality and disability-related costs are specific types of productivity losses, which are associated with a loss of earnings/time due to the disease. Cancer patients who cannot go back to work (in the labor market or in the household) due to disease-related disabilities incur additional indirect costs. Patients who eventually die of cancer face similar costs due to premature mortality. Therefore, younger patients usually incurred higher premature mortality costs due to overall productivity losses [20]. Four studies estimated these costs. Two studies concluded that males faced higher average short- and long-term disability-related costs relative to females $(\$ 66$ million vs $\$ 51$ million, and $\$ 369$ million vs 282 million, respectively) [20]. A study in Quebec estimated an annual indirect cost associated with cancer-related mortality at around $\$ 2.98$ billion [14]. Another study estimated an annual cost of $\$ 606$ million due to premature mortality, and $\$ 11.5$ million due to morbidity caused by melanoma at a national level [21]. Finally, the indirect cost associated with premature cancer-related mortality attributable to tobacco was estimated at $\$ 2.4$ billion for males and $\$ 1.7$ billion for females at the national level [20].

\section{Friction costs}

Friction costs were estimated in three studies. The friction cost approach defined friction costs as the costs and time that employers incur to replace workers who become absent due to cancer and to train the new workers who replace them [32]. In Quebec, the total friction cost faced by employers was estimated to be about $\$ 88.4$ million per year [14]. This cost accounted for the chain effect of job vacancies and friction time that occur as people shift between positions as they become available. Other studies found an annual friction cost of $\$ 23,987$ per patient among a bladder cancer population [19], and $\$ 6400$ per patient with lung cancer [27].

7. Societal productivity losses

Finally, five studies estimated productivity losses at the societal level. Unlike the previous categories, these estimates accounted for different types of indirect costs, such as lost productivity and home productivity losses in a single value. The overall productivity loss to society represents a multidimensional and more comprehensive measure of the indirect cost. The annual productivity 
loss due to a decrease in labor market participation associated with cancer was estimated at \$317 million in Quebec [14]. The same cost was estimated to be around $\$ 157$ million in Ontario among lung cancer patients [26]. Lastly, Wranik et al. estimated total productivity losses in Canada due to cancer from 1993 to 2013 and found an average cost of $\$ 75$ million per year [30].

\subsection{Approaches to Measuring Indirect Costs}

Productive labor market costs: All studies, which estimated work productive labor market costs, employed the human capital approach [33]. To obtain total and per-patient indirect cost estimates, some studies used a setting-specific average wage to assign a monetary value to the time lost from work and multiplied it by the overall time lost attributed to cancer. Other studies estimated patient-level costs based on the individual reported wages from tax files, surveys, or interviews. Three studies also employed the friction cost approach to estimate the short-term costs that employers incur due to absenteeism (staying home while sick) of cancer patients and their caregivers $[14,19,27]$. These costs pertain to the time required by employers to recruit and train new workers.

Productive non-labor market costs: These costs pertain exclusively to activities that are not related to labor market activities. Studies that reported on these costs also used the human capital approach to assign a monetary value to the leisure time lost, the time spent caring for patients, home production losses, and unpaid work.

\subsection{Quality Assessment}

The risk of bias assessment of retrospective and prospective studies is presented in Figure 2 and Appendix 4. While more than half of the studies had a low risk of bias, the studies with unclear and high-risk of bias usually failed to provide a thorough description of patient recruitment or the tools utilized to measure and report the outcome of interest. Overall, $60 \%$ of the retrospective studies had a low risk of bias, mainly due to a selection of an adequate and representative sample. Most of these studies employed administrative databases that allowed the identification of large samples that are usually generalizable. Furthermore, some of these studies with low risk of bias defined a control group of non-cancer patients to estimate incremental costs rather than net costs. They also employed various regression and statistical analyses that allowed controlling for potential confounder variables to further explain how different factors might be associated with indirect costs. However, $40 \%$ of the studies failed to provide a detailed and adequate methodology to select a representative sample size. Since the comparability and outcome dimensions were complete and appropriately defined, these studies were identified as having unclear risk of bias. No cross-sectional study had a high risk of bias. A similar proportion of prospective cohort studies had a low risk of bias (57\%). These studies adequately ensured that most patients were adequately followed-up and that the outcome assessment of the indirect cost burden had well-defined time horizons. Studies with unclear (29\%) and high risk of bias (14\%) usually lacked a description of the

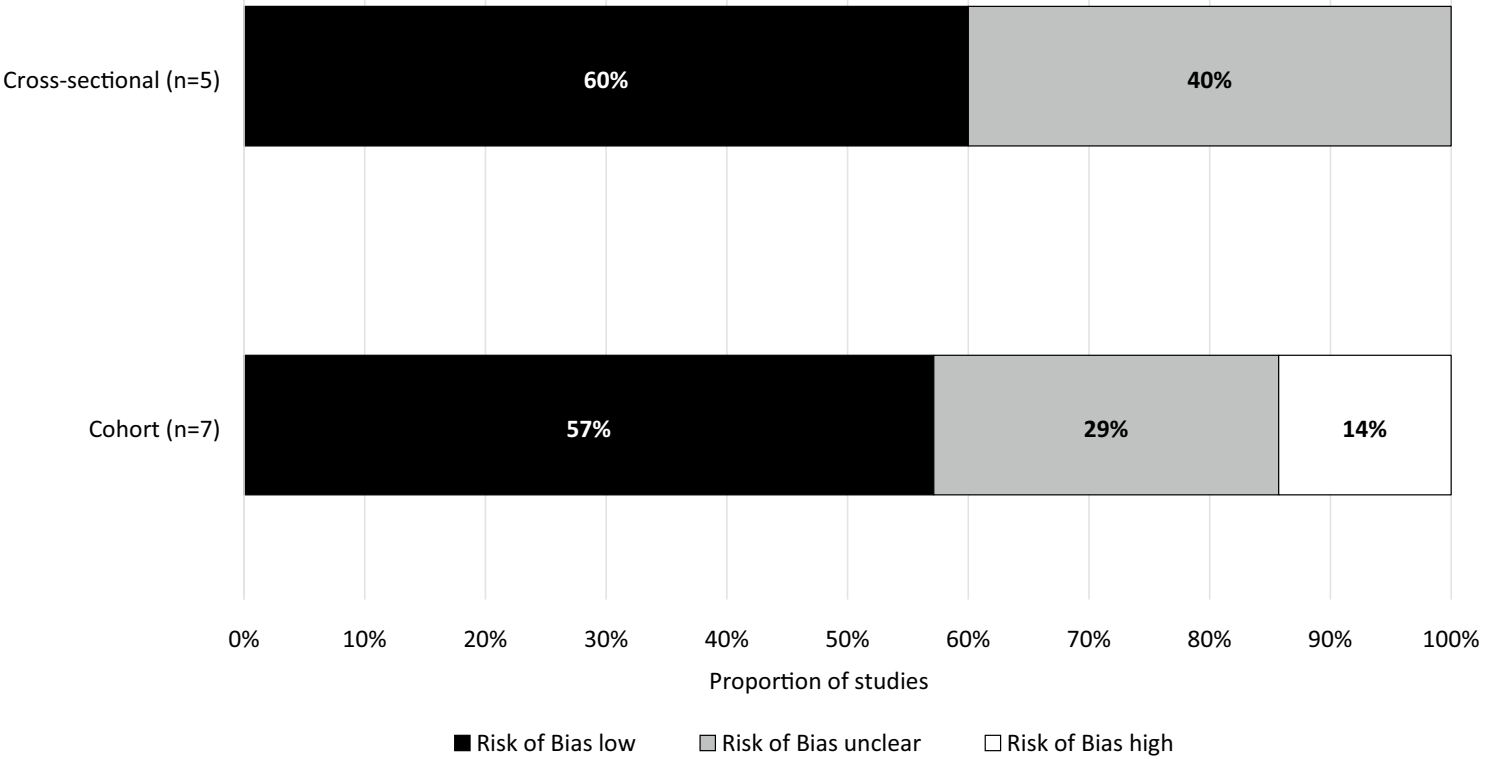

Fig. 2 Quality assessment of individual studies using the Newcastle-Ottawa Assessment Tool 
recruitment process to ensure representativeness of the sample or lacked a thorough description of how the outcomes were assessed (i.e. self-reports).

\section{Discussion}

This study presents an up-to-date and comprehensive review of the most recent literature on the indirect cost burden of cancer in Canada. We systematically reviewed studies that estimated labor and non-labor market-related costs, ranging from lost earnings to household productivity losses. The evidence suggests that cancer patients and their caregivers face a substantial indirect cost burden due to premature mortality and short- and long-term disabilities. Most of the reported costs were work-related productivity losses, rising to $\$ 80,000$ per patient per year. Leisure time costs and home production losses were also considerable, with maximum estimates of $\$ 18,000$ and $\$ 240,000$ per patient per year, respectively. Friction costs borne by employers were estimated at around $\$ 24,000$ per patient per year. Annual productivity losses at the societal level ranged from $\$ 75$ million in Nova Scotia to $\$ 317$ million in Quebec. We also found that caregivers of pediatric patients, women, younger patients, and those who were self-employed tended to face higher indirect costs.

A previous report by the Public Health Agency of Canada (PHAC) used a prevalence-based approach to evaluate the economic burden of chronic conditions between 2005 and 2008 , and estimated the indirect cost of cancer to be around $\$ 586,000,000$ in 2008 [8]. However, this value was likely underestimated, since the definition of the indirect cost burden was limited to the production losses due to illness, injury or premature death [8]. Other costs associated with nonlabor market activities, such as leisure time and informal caregiving, were not considered. Similarly, Hopkins et al. conducted a multi-database literature search on the national wage loss from cancer in Canada [9]. The studies identified in this review also limited their analyses to work-related costs. Our results provide an up-to-date and complete review of the indirect cost burden of cancer in Canada, by including literature published within the last 15 years and non-marketrelated costs, which account for a considerable portion of the burden. This review highlights the importance of ensuring that future reports and reviews reflect a more comprehensive assessment of the indirect burden of cancer to better account for all of the dimensions identified in this study in Canada

Two methods were identified in the literature to measure indirect costs - the human capital and the friction cost approaches. The human capital methodology applies average wages by sex and age to estimate the value of the time lost, usually from a labor market perspective [33]. Even though some indirect costs are not explicitly linked with workplace productivity (e.g. leisure time, or home productivity losses), the human capital estimation method allows assigning a monetary value to time (however defined). On the other hand, the friction cost approach assumes that work-related productivity losses are expected to be mitigated due to the eventual replacement of absentees [32]. As such, this theory suggests that in the long run, the society will adapt and reach the original production level as patients who leave the workforce are eventually replaced after an adaptation period. The costs associated with this short-term cycle are defined as friction costs. Some authors suggest that the friction cost method yields more accurate estimates compared to the human capital approach [8]. This is because friction costs do not overestimate the productivity losses by only considering the time it takes for society to replace the cases of absentees instead of assuming permanent vacancies. However, the friction cost approach usually neglects non-productive time (e.g. household production, leisure time) [33]. Although the human capital approach can be used to estimate non-market activity costs, the underlying assumptions need to be questioned to produce more nuanced and useful estimates. In particular, using a median wage to estimate lost wages due to cancer as opposed to actual wages could produce estimates that can be generalized across a broader population, which includes people who work at low-wage jobs. There are inherent assumptions about the value of time involved in the human capital approach, namely that the costs to individuals, families, and societies are higher if individuals earn higher wages; however, the burden is often highest for those with lower incomes. In other words, there are ethical implications to choices surrounding how to value indirect costs across a population.

We identified a trade-off between collecting primary data from patients and family members prospectively and using cross-sectional and longitudinal databases. Results from studies employing large administrative datasets are more easily generalizable to the overall population (if selected appropriately) and allow for the collection of individuallevel cost data for those who are represented therein. However, they have inherent limitations associated with the lack of quality controls and missing data issues. Prospective studies, on the other hand, allow for more precise data collection and, therefore, a more nuanced understanding of how cancer affects families across the care continuum. But these studies tend to be smaller in scale and may not address selection issues; their data collection may also be burdensome to patients and families. As such, it is fundamental to acknowledge the different types of data sources to understand the different ways in which the indirect cost burden can be reported. Although they provide an estimate of the indirect cost burden at different scales, both the micro (i.e. patient) and macro (i.e. societal) perspectives must be synthesized and understood to adequately shape policy focused towards reducing this burden. This two-pronged approach 
will provide a more comprehensive picture of the full indirect cost burden that individuals and society incur.

\subsection{Limitations}

There were some limitations associated with the review. By limiting our search to studies published after 2006, we may have missed relevant papers and reports, grey literature, or nonacademic references that the previous reviews failed to capture. There were also limitations with the evidence identified. Although cost estimates can be useful in directing resources for programming, cancer care is highly variable according to clinical factors such as tumor site, stage of cancer care, and stage of diagnosis; population demographics; geographic location; and coverage of oral chemotherapy and other types of medical and non-medical costs. Therefore, while aggregated cost estimates may help raise awareness of the overall cancer burden and allow for comparisons, greater efforts are needed to showcase the particular relevance to policy or programming to help address needs and barriers. Key clinical and population variables to consider across all cost areas include cancer survival probability and curative intent (vs palliative care), stage of diagnosis and stage of life at diagnosis, the level of invasiveness of treatment and the consequences of treatment interventions, and variability in access by different populations to cancer treatment. Therefore, there is a need for stratified cost estimates by cancer site, patient groups, and the cancer care continuum. Furthermore, future work should focus on understanding the provision of support and access to resources across different settings, provinces, and workplaces, which are intended to help mitigate indirect costs.

\section{Conclusion}

This review makes an important contribution to the literature on the economic burden of cancer in Canada, in particular the indirect burden. Measuring and describing the indirect costs faced by cancer patients and their caregivers allows for a better understanding of the different types of productivity losses and time costs. Once the different categories of the indirect costs are understood, it is important to consider ways to integrate these inputs in economic evaluations to get a better picture of the actual burden of illness from different perspectives. For instance, when indirect costs to patients (e.g. missed days of work while hospitalized) are accounted for in economic evaluations, drug expenses could be considered to be cost effective. However, work-related productivity losses are not the only indirect costs that should be considered when conducting economic evaluations from a more comprehensive societal perspective. According to the guidelines for the economic evaluation of health technologies in Canada from the Canadian Agency for Drugs and Technologies in Health $(\mathrm{CADTH})$, when conducting an evaluation from a societal perspective, all relevant costs to the patients, caregivers, and employers must be considered (e.g. patient out-of-pocket costs, patient time, lost productivity, home production costs, friction costs) [34]. This review suggests that the work productivity losses and the non-labor-market costs are considerable and should be considered in order to adequately measure the indirect burden from a societal perspective. These results will also help support a research agenda to fill the knowledge gaps with respect to direct and indirect costs of cancer across provinces and territories, and population sub-groups in Canada.

Author contributions Conception or design of work (NI, CdO, NF, BE). Data collection (NI, BE). Data analysis and interpretation (NI, $\mathrm{CdO}, \mathrm{NF}, \mathrm{BE})$. Drafting the article (NI, BE). Critical revision of the article (NI, CdO, NF, BE). Final approval for publication (NI, CdO, $\mathrm{NF}, \mathrm{BE})$.

Availability of data and material All data generated or analyzed during this study are included in this published article [and its supplementary information files].

\section{Declarations}

Funding None.

Conflict of interest The authors declare that they have no competing interests.

Ethics approval Not applicable.

Consent to participate Not applicable.

Consent for publication Not applicable.

Code availability Not applicable.

Open Access This article is licensed under a Creative Commons Attribution-NonCommercial 4.0 International License, which permits any non-commercial use, sharing, adaptation, distribution and reproduction in any medium or format, as long as you give appropriate credit to the original author(s) and the source, provide a link to the Creative Commons licence, and indicate if changes were made. The images or other third party material in this article are included in the article's Creative Commons licence, unless indicated otherwise in a credit line to the material. If material is not included in the article's Creative Commons licence and your intended use is not permitted by statutory regulation or exceeds the permitted use, you will need to obtain permission directly from the copyright holder. To view a copy of this licence, visit http://creativecommons.org/ licenses/by-nc/4.0/. 


\section{References}

1. Canadian Cancer Statistics 2019. Canadian Cancer Society: Government of Canada; 2019.

2. Table 13-10-0394-01: leading causes of death, total population, by age group. Statistics Canada: Government of Canada; 2019.

3. Brenner DR, Weir HK, Demers AA, Ellison LF, Louzado C, Shaw A, et al. . Projected estimates of cancer in Canada in 2020. CMAJ. 2020;192(9):E199-205.

4. Pisu M, Azuero A, McNees P, Burkhardt J, Benz R, Meneses K. The out of pocket cost of breast cancer survivors: a review. J Cancer Surviv. 2010;4(3):202-9.

5. Jo C. Cost-of-illness studies: concepts, scopes, and methods. Clin Mol Hepatol. 2014;20(4):327-37.

6. de Oliveira C, Weir S, Rangrej J, Krahn MD, Mittmann N, Hoch JS, et al. . The economic burden of cancer care in Canada: a populationbased cost study. CMAJ Open. 2018;6(1):E1-10.

7. Yabroff KR, Lund J, Kepka D, Mariotto A. Economic burden of cancer in the United States: estimates, projections, and future research. Cancer Epidemiol Biomark Prev. 2011;20(10):2006-14.

8. Canada PHAo. Economic burden of illness in Canada 2005-2008. PHAC. 2014.

9. Hopkins RB, Goeree R, Longo CJ. Estimating the national wage loss from cancer in Canada. Curr Oncol. 2010;17(2):40-9.

10. Geiger AM, O'Mara AM, McCaskill-Stevens WJ, Adjei B, Tuovenin $\mathrm{P}$, Castro KM. Evolution of cancer care delivery research in the NCI Community Oncology Research Program. J Natl Cancer Inst. 2019;112(6):557-61.

11. Moher D, Liberati A, Tetzlaff J, Altman DG, Group P. Preferred reporting items for systematic reviews and meta-analyses: the PRISMA statement. PLoS Med. 2009;6(7):e1000097.

12. Wells G, Shea B, O'Connell D, Peterson J, Welch V, Losos M, et al. . The Newcastle-Ottawa Scale (NOS) for assessing the quality of nonrandomised studies in meta-analyses. The Hospital of Ottawa. 2019.

13. Bank of Canada's Inflation Calculator: Bank of Canada; 2020. https ://www.bankofcanada.ca/rates/related/inflation-calculator/. Accessed 3 May 2020

14. Boucher P, Beauregard H. Le coût économique du cancer au Québec, en 2008. Coalition Priorite Cancer au Quebec; 2008.

15. de Oliveira C, Bremner KE, Ni A, Alibhai SM, Laporte A, Krahn MD. Patient time and out-of-pocket costs for long-term prostate cancer survivors in Ontario, Canada. J Cancer Surviv. 2014;8(1):9-20.

16. Dumont S, Jacobs P, Turcotte V, Turcotte S, Johnston G. Palliative care costs in Canada: a descriptive comparison of studies of urban and rural patients near end of life. Palliat Med. 2015;29(10):908-17.

17. Jeon SH. The long-term effects of cancer on employment and earnings. Health Econ. 2017;26(5):671-84.

18. Jeon SH, Pohl RV. Health and work in the family: Evidence from spouses' cancer diagnoses. J Health Econ. 2017;52:1-18.

19. Jung YL, Tompa E, Longo C, Kalcevich C, Kim J, Song C, et al. . The economic burden of bladder cancer due to occupational exposure. J Occup Environ Med. 2018;60(3):217-25.
20. Krueger H, Andres EN, Koot JM, Reilly BD. The economic burden of cancers attributable to tobacco smoking, excess weight, alcohol use, and physical inactivity in Canada. Curr Oncol. 2016;23(4):241-9.

21. Krueger H, Williams D, Chomiak M, Trenaman L. The economic burden of skin cancer in Canada: current and projected. Can Partnersh Against Cancer; 2010. http://krueger.ca/wp-content/uploa ds/2015/08/skincancer.pdf. Accessed 30 April.

22. Lauzier S, Maunsell E, Drolet M, Coyle D, Hebert-Croteau N. Validity of information obtained from a method for estimating cancer costs from the perspective of patients and caregivers. Qual Life Res. 2010;19(2):177-89.

23. Lauzier S, Maunsell E, Drolet M, Coyle D, Hebert-Croteau N, Brisson $\mathrm{J}$, et al. . Wage losses in the year after breast cancer: extent and determinants among Canadian women. J Natl Cancer Inst. 2008;100(5):321-32.

24. Longo CJ, Fitch M, Deber RB, Williams AP. Financial and family burden associated with cancer treatment in Ontario, Canada. Support Care Cancer. 2006;14(11):1077-85.

25. Mofidi A, Tompa E, Spencer J, Kalcevich C, Peters CE, Kim J, et al. . The economic burden of occupational non-melanoma skin cancer due to solar radiation. J Occup Environ Hyg. 2018;15(6):481-91.

26. Smetanin P, Stiff D, Briante C, Ahmad S, Wong L, Ler A. Life and economic burden of lung disease in Ontario: 2011 to 2041. Risk Analytica, on behalf of the Ontario Lung Association; 2011.

27. Tompa E, Kalcevich C, McLeod C, Lebeau M, Song C, McLeod $\mathrm{K}$, et al. . The economic burden of lung cancer and mesothelioma due to occupational and para-occupational asbestos exposure. Occup Environ Med. 2017;74(11):816-22.

28. Tsimicalis A, Stevens B, Ungar WJ, Greenberg M, McKeever $\mathrm{P}$, Agha M, et al. . Determining the costs of families' support networks following a child's cancer diagnosis. Cancer Nurs. 2013;36(2):E8-19.

29. Tsimicalis A, Stevens B, Ungar WJ, McKeever P, Greenberg M, Agha M, et al. . A prospective study to determine the costs incurred by families of children newly diagnosed with cancer in Ontario. Psychooncology. 2012;21(10):1113-23.

30. Wranik WD, Muir A, Hu M. Costs of productivity loss due to occupational cancer in Canada: estimation using claims data from Workers' Compensation Boards. Health Econ Rev. 2017;7(1):9.

31. Yu M, Guerriere DN, Coyte PC. Societal costs of home and hospital end-of-life care for palliative care patients in Ontario, Canada. Health Soc Care Commun. 2015;23(6):605-18.

32. Brouwer WB, Koopmanschap MA. The friction-cost method : replacement for nothing and leisure for free? Pharmacoeconomics. 2005;23(2):105-11.

33. Pike J, Grosse SD. Friction cost estimates of productivity costs in cost-of-illness studies in comparison with human capital estimates: a review. Appl Health Econ Health Policy. 2018;16(6):765-78.

34. CADTH. Guidelines for the economic evaluation of health technologies: Canada. 4th edition; Ottawa; 2017.

\section{Affiliations}

\section{Nicolas Iragorri ${ }^{1,2,4}\left(\right.$ D $\cdot$ Claire de Oliveira ${ }^{1,2,3} \cdot$ Natalie Fitzgerald ${ }^{1} \cdot$ Beverley Essue ${ }^{1,2}$}

1 The Canadian Partnership Against Cancer, Toronto, ON, Canada

2 Institute of Health Policy, Management and Evaluation, Dalla Lana School of Public Health, The University of Toronto, Toronto, ON, Canada
3 Centre for Health Economics and Hull York Medical School, University of York, Heslington, York, UK

4 University of Toronto, 27 King's College Cir, Toronto, ON M5S, UK 